\title{
Ostwald-Driven Phase Separation In Bimetallic
}

\section{Nanoparticle Assemblies}

\author{
Geoffroy Prévot, ${ }^{\dagger}$ Nhat Tai Nguyen, ${ }^{\ddagger}$ Damien Alloyeau, ${ }^{\ddagger}$ Christian Ricolleau, ${ }^{\ddagger}$ Jaysen Nelayah ${ }^{\ddagger}$ \\ † Sorbonne Universités, UPMC Univ Paris 06, CNRS-UMR 7588, Institut des NanoSciences de \\ Paris, F-75005, Paris, France \\ † Laboratoire de Matériaux et Phénomènes Quantiques, Bâtiment Condorcet, CNRS UMR 7162, \\ Université Paris Diderot, Paris, France
}

\section{Details of thermodynamic calculations}

In this part, we derive a size-concentration relation for supported bimetallic A-B nanoparticles having a truncated spherical shape. We assume that the detachment rate for atoms of type A is much smaller than the one of atoms of type B and that the thermodynamic equilibrium is reached for B atoms.

The chemical potential of the B element is given by $\mu_{B}=\left.\frac{\partial G}{\partial N_{B}}\right|_{T, P, N_{A}}$. In a particle, neglecting the effects of the edges, the Gibbs free enthalpy is given by the sum of a bulk contribution and a surface term: 
$G=G_{\text {bulk }}+G_{\text {surf }}$

The bulk term of the Gibbs free enthalpy is given by:

$G_{\text {bulk }}=N_{\mathrm{A}} g_{0}^{\mathrm{A}}+N_{\mathrm{B}} g_{0}^{\mathrm{B}}+N g_{\text {mix }}$

where $N=N_{\mathrm{A}}+N_{B}$ is the number of atoms in the particle, and the mixing atomic free enthalpy is $g_{\text {mix }}=h_{\text {mix }}-T s_{\text {mix }}$. The mixing enthalpy $h_{\text {mix }}$ can be well approximated by: ${ }^{1}$

$h_{\text {mix }}=x(1-x)\left(h_{0}+h_{1}(1-2 x)+h_{2} x(1-x)\right)$

where $x=N_{\mathrm{B}} / N$, whereas the mixing entropy $s_{\text {mix }}$ is given by:

$s_{\text {mix }}=-k(x \ln (x)+(1-x) \ln (1-x))+s_{\mathrm{e}}$

$s_{\mathrm{e}}$ is the excess entropy that can also be approximated by: ${ }^{1}$

$s_{\mathrm{e}}=x(1-x)\left(s_{0}+s_{1}(1-2 x)+s_{2} x(1-x)\right)$

Thus, the Gibbs free enthalpy depends not only on the number of atoms in the particle but also on the composition, which varies when the number of atoms of one specie changes.

The surface term $G_{\text {surf }}$ is given by:

$G_{\text {surf }}=\gamma S+\left(\gamma_{\text {int }}-\gamma_{\text {sub }}\right) A$

where $\gamma, \gamma_{\text {int }}$, and $\gamma_{\text {sub }}$ are the surface free energy of the particle, the interfacial free energy and the surface free energy of the substrate. In the following, we suppose that they are all isotropic, which corresponds to the case of a liquid droplet on a surface. $S$ is the area of the particle in 
contact with vacuum, and $A$, the interfacial area. For a truncated sphere of radius $r$, the surface, volume and interfacial area are given by :

$$
\begin{aligned}
& S=2 \pi r^{2}(1-\cos (\theta)) \\
& V=\alpha r^{3} \\
& \text { with } \alpha=\frac{\pi}{3}\left(2-3 \cos (\theta)+\cos ^{3}(\theta)\right) \\
& A=\pi r^{2} \sin ^{2}(\theta)
\end{aligned}
$$

where $\theta$ is the contact angle between the particle and the substrate. At equilibrium, the contact angle is related to the surface free energies through the Young relation:

$$
\cos (\theta)=\frac{\gamma_{\mathrm{sub}}-\gamma_{\mathrm{int}}}{\gamma}
$$

Thus, $G_{\text {surf }}=\gamma S-\gamma A \cos (\theta)=2 \pi r^{2} \gamma\left(2-3 \cos (\theta)+\cos ^{3}(\theta)\right)$, or:

$$
G_{\text {surf }}=3 \gamma \alpha(N \Omega)^{2 / 3}
$$

where $\Omega$ is the mean atomic volume.

In the following, we define $\Delta \mu_{\mathrm{B}}(T, N, x)=\mu_{\mathrm{B}}(T, N, x)-\mu_{B}^{0}(T)$ as the difference of chemical potential of element B between a bimetallic A-B nanoparticle and the pure bulk material.

$\Delta \mu_{\mathrm{B}}(T, N, x)$ can be decomposed into a mixing and a surface contribution:

$$
\Delta \mu_{\mathrm{B}}(T, N, x)=\mu_{\mathrm{B}, \operatorname{mix}}(T, x)+\mu_{\mathrm{B}, \operatorname{surf}}(T, N, x)
$$


with $\quad \mu_{\mathrm{B}, \text { mix }}(T, x)=\left.\frac{\partial N g_{\text {mix }}}{\partial N_{\mathrm{B}}}\right|_{T, P, N_{A}}$ and $\mu_{\mathrm{B}, \text { surf }}(T, N, x)=\left.\frac{\partial G_{\text {surf }}}{\partial N_{\mathrm{B}}}\right|_{T, P, N_{A}}$. For a nanoparticle, contrary to what could be observed for bulk material, one has to take into account the fact that when the number of $\mathrm{B}$ atoms varies, the composition also varies.

$$
\left.\frac{d x}{d N_{\mathrm{B}}}\right|_{N_{A}}=\frac{1-x}{N}
$$

Deriving equations (S2-S4) gives:

$\mu_{\mathrm{B}, \text { mix }}(T, x)=(1-x)^{2}\left(g_{0}+g_{1}(1-4 x)+g_{2} x(2-3 x)\right)+k T \ln (x)$

and deriving equation (S8) gives rise to:

$\mu_{\mathrm{B}, \mathrm{surf}}(T, N, x)=\frac{\tilde{\gamma}(x)}{N^{1 / 3}}$

with $\tilde{\gamma}=2 \gamma \alpha^{1 / 3} \Omega^{2 / 3}+3(1-x) \frac{\partial}{\partial x}\left(\gamma \alpha^{1 / 3} \Omega^{2 / 3}\right)_{N}$

Eq. (S12) can also be written as $\mu_{\mathrm{B}, \text { surf }}(T, N, x)=2 \frac{\gamma \Omega}{r}+\left.3(1-x) \frac{\partial}{\partial x} \frac{\gamma \Omega}{r}\right|_{N}$. The first term is the classical Laplace pressure, whereas the second term is related to the dependence of $\gamma, \theta$, and $\Omega$ with $x$, for a given value of $N$.

The chemical potential is thus:

$$
\Delta \mu_{\mathrm{B}}(T, N, x)=\widetilde{\gamma}(x) / N^{1 / 3}+\mu_{\mathrm{B}, \mathrm{mix}}(T, x)
$$


At the thermodynamic equilibrium, $\Delta \mu_{\mathrm{B}}$ is constant. The particle radius and concentration are thus related through:

$$
r=\left(\frac{\Omega}{\alpha}\right)^{1 / 3} \frac{\tilde{\gamma}(x)}{\Delta \mu_{\mathrm{B}}-\mu_{\mathrm{B}, \text { mix }}(T, x)}
$$

\section{Details of the kinetic Monte-Carlo (kMC) simulations}

Here, we briefly recall the main features of the $\mathrm{kMC}$ algorithm. The algorithm simulates the thermal evolution of $3 \mathrm{D}$ particles on an atomically flat homogeneous surface where periodic boundary conditions are applied, through exchange of atoms. Particles are considered to be isotropic, unstrained and locally at equilibrium, and have a truncated spherical shape, of radius $r$, with a contact angle $\theta$. In the surface plane, the footprint of the particles is thus a disk of size $r_{D}=r \sin (\theta)$. The effect of islands reshaping has been discussed by Shorlin et al., for the case where the detachment and attachment rates are high enough to induce a shape variation of the particle with growth rate. Only particles having their radius close to the equilibrium radius adopt an equilibrium shape. ${ }^{2}$ Here, in order to speed up the algorithm, we consider only the variation of size of the particles, and we assume that their shape remain unchanged.

Each Monte-Carlo step consists of a three stage atomic motion: detachment of an atom from a particle, diffusion on the surface, attachment to a particle. The rate of detachment is given by $v_{d} \exp \left(-E_{d} / k T\right) \exp \left(-\Delta E-E_{a} / k T\right)$ where $v_{d}$ is an attempt frequency, $E_{d}$ an energy barrier, $\Delta E$ is the energy difference of the nanoparticle before and after detachment of an atom and $E_{a}$ is the adsorption energy of the corresponding atom on the substrate. In the simulation, we have 
used the theoretical values of the adsorption energies of $\mathrm{Au}$ and $\mathrm{Pd}$ on graphene: $E_{a}(\mathrm{Pd})=$ $1.081 \mathrm{eV}, \quad E_{a}(\mathrm{Au})=-0.096 \mathrm{eV}$ and the corresponding height barriers for diffusion $E_{d}(\mathrm{Pd})=0.037 \mathrm{eV}$ and $E_{d}(\mathrm{Au})=0.007 \mathrm{eV} .^{3}$ In order to roughly reproduce the timescale of the experiments, the value of $v_{d}$ has been fixed to $10^{16} \mathrm{~Hz}$ for both metals. This is a high frequency as compared to what is generally observed $\left(10^{13} \mathrm{~Hz}\right)$. This could reflects that adsorption energies on amorphous carbon are lower than the theoretical values computed for graphene. $\Delta E$ is given by the cohesive and surface energy of the particle (see thermodynamics). After detachment, diffusion trajectories are not explicitly computed. Taking into account the local environment of a particle, simplified trajectories are performed instead of single atomic diffusion events, that considerably speed-up the algorithm, while preserving the distribution of attachment probability on the surrounding particles. A diffusing atom attach to an island as soon as it reaches its periphery. No kinetic barrier for attachment has been considered.

Atoms diffuse, thus, one by one and, except for the island which loses an atom, no other evolution of the island assembly is taken into account during atomic diffusion. We use the BKL algorithm for definition of the timescale: at each step, the time-step is the inverse of the sum of the probabilities of all detachment events. A random number is then generated and used for choosing a possible atomic detachment accordingly to its probability. Using this approach, we assume that the duration of the atomic diffusion is much smaller than the time-step, i.e. the interval between two detachment events from the islands. In particular, we neglect the fact that two adatoms could meet and form a new island. For metallic clusters on carbon, this assumption is valid since the energy barrier for atomic detachment from particles is much higher than the energy barrier for atomic diffusion on the substrate. 
Figure S1 shows the evolution of the chemical potential for $\mathrm{Au}$ and $\mathrm{Pd}$ in the particles after 10h annealing at $873 \mathrm{~K}$, for $\gamma_{A u}=9.4 \mathrm{eV} \cdot \mathrm{nm}^{-2}$ and $\gamma_{P d}=28 \mathrm{eV} \cdot \mathrm{nm}^{-2}$. Whereas the Au chemical potential strongly varies with size, the Pd chemical potential is nearly constant.

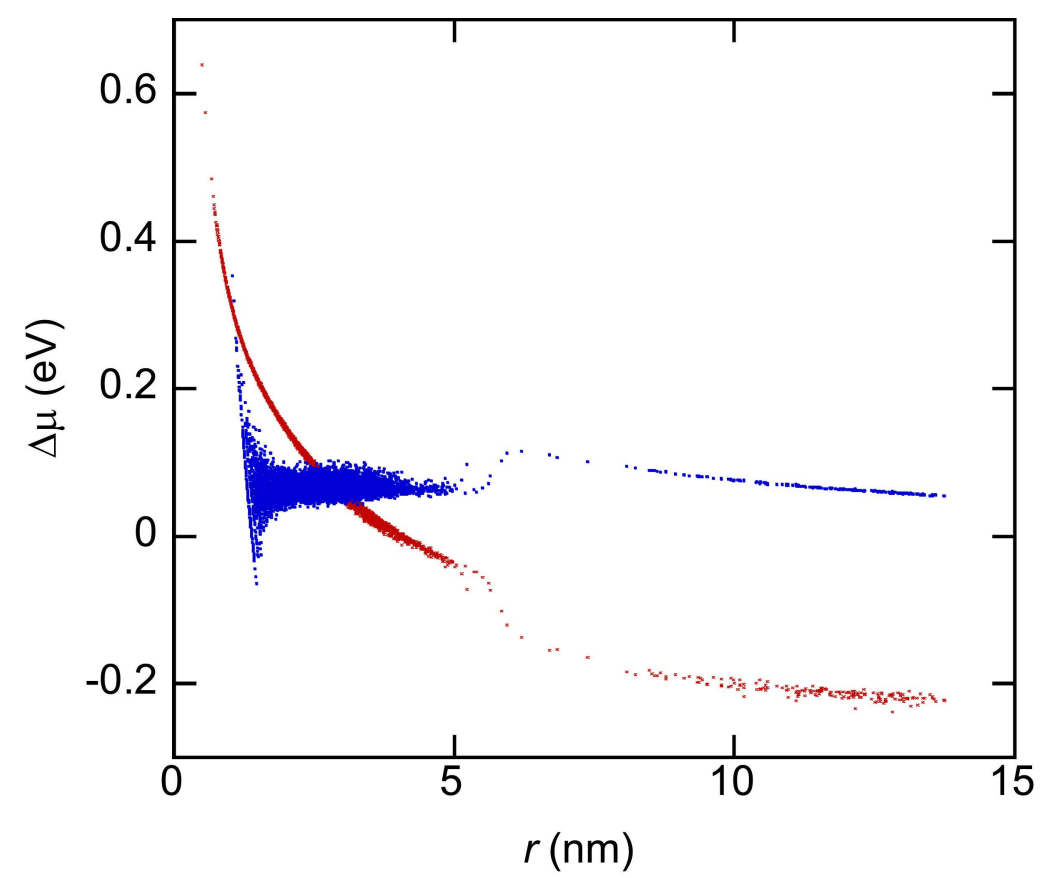

Figure S1: Variation of the chemical potential in the NPs as a function of their radius $r$, obtained from a kMC simulation of $10 \mathrm{~h}$ annealing at $873 \mathrm{~K}$, for $\gamma_{P d}=9.4 \mathrm{eV} \cdot \mathrm{nm}^{-2}$ and $\gamma_{P d}=28 \mathrm{eV} . \mathrm{nm}^{-2}$. Red crosses: Au chemical potential. Blue dots: Pd chemical potential.

(1) Williams, R. O. Parameterization of the Solution Data for Binary Alloys by Hultgren et al. Calphad 1991, 15, 1-10.

(2) Shorlin, K.; Krylov, S.; Zinke-Allmang, M. Fundamental Problems Concerning Three Dimensional Clustering on Surfaces. Phys. A 1998, 261, 248-265.

(3) Chan, K. T.; Neaton, J. B.; Cohen, M. L. First-Principles Study of Metal Adatom Adsorption on Graphene. Phys. Rev. B 2008, 77, 235430. 